\title{
Bus to House: Voice \& Touch Based Dynamic Bus Navigation System Based On Zigbee, Iot \& Android
}

\author{
K.Rakshambighai ${ }^{1}$,A.G.Saranya ${ }^{2}$,M.Maheswari ${ }^{3}$ \\ ${ }^{1}$ IV CSE student [e-mail: rakshu.rsg@gmail.com] \\ ${ }^{2}$ IV CSE student [e-mail: saranyagovindan2@gmail.com] \\ ${ }^{3}$ Associate professor, [e-mail:m.mahe05@gmail.com]
}

\begin{abstract}
Many transportation modes are available in our country but, still transportation is a major issue nowadays in metropolitan cities and urban areas. Increase in number of cars on road leads to many problems such as traffic congestion, health problems, air pollution, environmental degradation etc. This problem can be reduced by using a mechanism called as "Bus sharing or pooling". The aim of the project is to "get Buses into passenger's houses as Call taxi is functioning". This system will avoid traditional route of the buses. Bus pooling is more efficient because of reduces each travelling cost, fuel cost, stress of driving and security to passengers etc.
\end{abstract}

Keywords: Autonomous Vehicle,Dynamic Bus Navigation, Zigbee

\section{Introduction}

GPS provides specially coded satellite signals that can be processed in a GPS receiver, enabling the receiver to compute position, velocity and time. Four GPS satellite signals are used to compute positions in three dimensions and the time offset in the receiver clock. GPS is being installed in vehicles with the primary objective of providing accurate monitoring of location. To provide better customer service in the competitive $\mathrm{Cab}$ business, fast and efficient dispatching is a critical factor. Most cab dispatching systems rely on good staff and teamwork, but misunderstandings occur when bookings are transmitted by voice. This project will initiate a new application using GPS, a smart satellite-based system for tracking and dispatching Cabs to commuters. With the new system, the nearest Cab is located with the help of GPS and then bookings and routings are transmitted to the Cab drivers display unit in digital form. Through the use of this computerized satellite-based $\mathrm{Cab}$ dispatching and data network system, operational errors and costs are expected to be reduced. With this system, computerized dispatching links both commuters and Cab drivers by computer and lets the operators know, by satellites, where their Cabs are located.

Every $\mathrm{Cab}$ has its location updated periodically. The system can find the nearest vacant $\mathrm{Cab}$, and the Cab's display panel can tell the driver passenger's location. Once a job is acknowledged by the driver, the system can automatically contact the customer telling him or her the Cab's license plate number through a synthesized text message. In order to make the whole system fully autonomous, the project is also implemented on an autonomous robot equipped with GPS and Compass module which has the ability to navigate between a series of way points to reach its destined coordinates.

In the EXISTING SYSTEM, there is no tracking of Buses happening. GPS based Vehicle is only the solution but still arrival Timing of the Buses are not intimated to the bus stop. In the PROPOSED MODEL, GPS Hardware is attached in every bus for the continuous tracking of the vehicle. The most important part of this project is to get Buses into passenger's houses as Call taxi is functioning. This system will avoid traditional route of the buses. The MODIFICATION Process is our implementation. We deploy an android based application on all customers' mobile and customer can book the bus from their android application either through normal touch based or by Voice. Voice based booking is helpful for the visually impaired people. One Zigbee is attached with the bus and another Zigbee is attached with the server. Android user requests the query specifying Source $\&$ the destination to the server. Server will verify the nearest available bus analyzing through buses mobility status via Zigbee. If there is no request for a particular stop bus will dynamically reroutes to another shortest route without crossing that stop.

The Central Control Room (CCR) controller consists of Arduino Uno and GSM shield, the programming of main controller has been done in such a way that it initially waits to receive the text message. Numbers of both Cabs have already been stored in main controller and as the main controller receives the SMS it will perform a comparison to detect whether this SMS is from Cabs or user. If the Cab is vacant, then driver will press the vacant button, the main controller will receive the latest coordinates of the Cab and also the main controller will update the status of $\mathrm{Cab}$ as vacant, after that the $\mathrm{CAB}$ will automatically updates its coordinates to the main controller every 10 seconds. The user will send his coordinates using his/her Smartphone application, the main controller will look for the number first and if this number is not either of the Cab then it 
Bus to House: Voice \& Touch Based Dynamic Bus Navigation System Based On Zigbee, Iot \& ..

will save these coordinates as the user's coordinates. After number detection main controller will look for the all the possible Cabs and directs the nearest possible Cab towards user. The flowchart of main controller after receiving request from user.

Cab and User: If the $\mathrm{Cab}$ is available and the user has send the requests for the requests for $\mathrm{Cab}$, the Central Control Room (CCR) send the user's coordinates to the nearest $\mathrm{Cab}$ and also the CCR will send the confirmatory message (Your CAB has been directed to your present location) to the user, and after that the CCR will up the status of $\mathrm{Cab}$ as non vacant and the Cab will be guided by GPS towards the user using python language.

No vacant Cab only user: If there is no vacant $\mathrm{Cab}$ available and user send request of $\mathrm{Cab}$ to the CCR using Smartphone app, then CCR will send a busy text (Sorry, No vacant CABS are available.) to the user.

\section{Related Work}

Albert Y.S. Lam, Yiu-Wing Leung, and Xiaowen Chu et al [1] proposed that Technology of autonomous vehicles (AVs) is getting mature and many $\mathrm{AVs}$ will appear on the roads in the near future. AVs become connected with the support of various vehicular communication technologies and they possess high degree of control to respond to instantaneous situations cooperatively with high efficiency and flexibility. In this paper, we propose a new public transportation system based on AVs. It manages a fleet of AVs to accommodate transportation requests, offering point to- point services with ride sharing. We focus on the two major problems of the system: scheduling and admission control. The former is to configure the most economical schedules and routes for the AVs to satisfy the admissible requests while the latter isto determine the set of admissible requests among all requests to produce maximum profit. The scheduling problem is formulated as a mixed integer linear program and the admission control problem is cast as a bi level optimization, which embeds the scheduling problem as the major constraint. By utilizing the analytical properties of the problem, we develop an effective genetic-algorithm-based method to tackle the admission control problem. We validate the performance of the algorithm with real-world transportation service data.

Broggi $^{1}$, L. Bombini ${ }^{1}$, S. Cattani ${ }^{1}$, P. Cerri ${ }^{1}$, and R.I. Fedriga ${ }^{1}$ et al [2] proposed a new model in which the design issues that were considered for the equipment of 4 identical autonomous vehicles that will drive themselves without human intervention on an intercontinental route for more than $13,000 \mathrm{~km}$. Autonomous vehicles have been demonstrated able to reach the end of a 220 miles off-road trail (in the DARPA Grand Challenge), to negotiate traffic and obey traffic rules (in the DARPA Urban Challenge), but no one ever tested their capabilities on a long, intercontinental trip and stressed their systems for 3 months in a row. This paper presents the technological challenge of a set of vehicles that will run the VisLab Intercontinental Autonomous Challenge (VIAC). The challenge is scheduled to take place during the 2010 World Expo in Shanghai, China. Being currently under preparation, this paper focuses on the development, the vehicles' technical details, and the challenge itself. Other following papers will describe the outcome of the challenge and its results.

Kailong Zhang, Member, IEEE, Dafang Zhang, Arnaud de La Fortelle, Member, IEEE, Xiao Wu, and Jean Grégoire et al [3] proposed that Scheduling driverless vehicles with different priorities to pass through intersections efficiently and safely has been becoming an important passing-through intersection (PTI) problem in the field of novel intelligent traffic systems (ITS), which is increasingly becoming cyber-physicalfused and social-service oriented. Considering new emerging features with possible priorities, a novel centralized priority scheduling mechanism is mainly explored in this paper. First, related pivotal aspects of environment and driverless vehicles are modeled by fusing their physical and kinematic characters. Based on these models, PTI-related motions are further abstracted as several reservation-oriented standard states and actions. Then, an event-triggered and state-driven autonomous control procedure is designed. By mapping vehicular relations in spatiotemporal domain into time-distance windows, a universal passing-through principle, rules, and priority-based scheduling mechanisms are proposed and described in detail. Finally, a priority scheduling algorithm sPriorFIFO is proposed and designed. These models and mechanisms are then implemented within an algorithm simulator, through which scheduling performances are verified and evaluated

Plamen Petrov and Fawzi Nashashibi et al [4] In this paper, we present a nonlinear adaptive controller for a two-vehicle automated overtaking maneuver. We consider the problem of an autonomous threephase overtaking without the use of any roadway marking scheme or inter-vehicle communication. The developed feedback controller requires information for the current relative inter vehicle position and orientation available from onboard sensors only. We apply standard robotic nomenclature for translational and rotational displacements and velocities and propose a general kinematic model of the vehicles during the overtaking maneuver including for the relative inter-vehicle kinematics. The overtaking maneuver is investigated as a tracking problem with respect to desired polynomial virtual trajectories for every phase, which are generated in real time. An update control law for the automated overtaking vehicle is designed that allows tracking the desired trajectories in the presence of unknown velocity of the overtaken vehicle. 
Andrei Furda1, Laurent Bouraoui2, Michel Parent2, and Ljubo Vlacic1 et al [5] This paper elaborates on the Cybercars-2 Wireless Communication Framework for driverless city vehicles, which is used for Vehicle-to-Vehicle and Vehicle-to-Infrastructure communication. The developed framework improves the safety and efficiency of driverless city vehicles. Furthermore, this paper also elaborates on the vehicle control software architecture. On road tests of both the communication framework and its application for real-time decision making show that the communication framework is reliable and useful for improving the safe operation of driverless city vehicles.

Qingquan Li, Long Chen, Ming Li, Shih-Lung Shaw and Andreas N“uchter et al [6] proposes that Autonomous vehicle navigation is challenging since various types of road scenarios in real urban environments have to be considered, especially when only perception sensors are used, without position information. This paper presents a novel real-time optimal-drivable-region and lane detection system for autonomous driving based on the fusion of Light Detection and Ranging (LIDAR) and vision data. Our system uses a multisensory scheme to cover the most drivable areas in front of the vehicle. We propose a feature-level fusion method for LIDAR and vision data and an optimal selection strategy for detection of the best drivable region. Then a conditional lane detection algorithm is selectively executed depending on an automatic classification of the optimal drivable region. Our system successfully handles both structured and unstructured roads. The results of several experiments are provided to demonstrate the reliability, effectiveness, and robustness of the system.

\section{Existing System:}

In the EXISTING SYSTEM, there is no tracking of Buses happening. GPS based Vehicle is only the solution but still arrival Timing of the Buses are not intimated to the bus stop. The disadvantage of the existing system is time consuming process and unreliable For a short distance travelling cab cost is very high. There is huge problem of traffic on roads these days and the increase in fuel prices add to the misery of daily users of personal vehicles.

\section{Proposed System:}

In the PROPOSED MODEL, GPS Hardware is attached in every bus for the continuous tracking of the vehicle. The most important part of this project is to get Buses into passenger's houses as Call taxi is functioning. This system will avoid traditional route of the buses. One Zigbee is attached with the bus and another Zigbee is attached with the server. Android user requests the query specifying Source \& the destination to the server. Server will verify the nearest available bus analyzing through buses mobility status via Zigbee.In our implementation IR sensor will indicate the bus arrival in the stop and ultrasonic sensor will detect the obstacles. Motor and its driver represent the bus section. Based on the request, bus will move around the specific path by automatically detecting the IR sensor and obstacle.

The advantage is it is an less time consuming and reliable process.

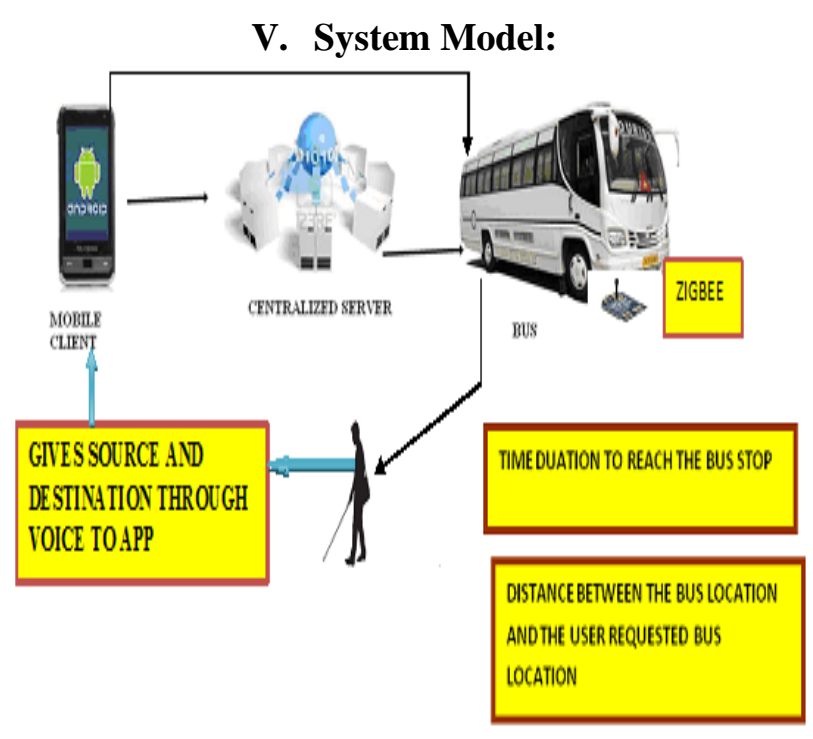

\section{A. Server Deployment:}

In this module we will develop server with the application and database related to available buses and routes of the area. Server will collect the input request from the user and the process the request with available route and bus stops the reply to the user and also bus driver 


\section{B. Android Application:}

In this module android application is developed to access the server for the bus request. From the android application the passenger will give the source and destination places of the bus stops. Then the android application forwards the request to server. Server will process the requests with available information.

\section{User Input Analysis:}

In this module user input is processed in the server. Based on the source and destination server will verifies the route details, bus information and also bus timings of the particular bus stops. If anybody is there in particular bus stops Server will intimates it to the bus driver about bus stop and also route. Otherwise driver can reroute the bus with alternate route.

\section{Embedded Hardware:}

In this module we will fabricates embedded kit to show the demo of the bus movements in the specified routes. From the server system to request will forward to that particular bus through zigbee communication. Based on the request bus kit will moved to the specified routes.

\section{E. Dynamic Route Processing:}

Based on the User request server will process the route dynamically. So that the driver will moves the bus to that specified routes.

With this dynamic way we can analyse the correct route to minimise the time and petrol cost also.

\section{E. Intimation Of Nearest Bus:}

If there is any buses is not there in that particular route means server will check that if there any other buses available in the nearest routes. So that it will intimate passenger details to that bus driver to pick up the passenger.

\section{Bus Module:}

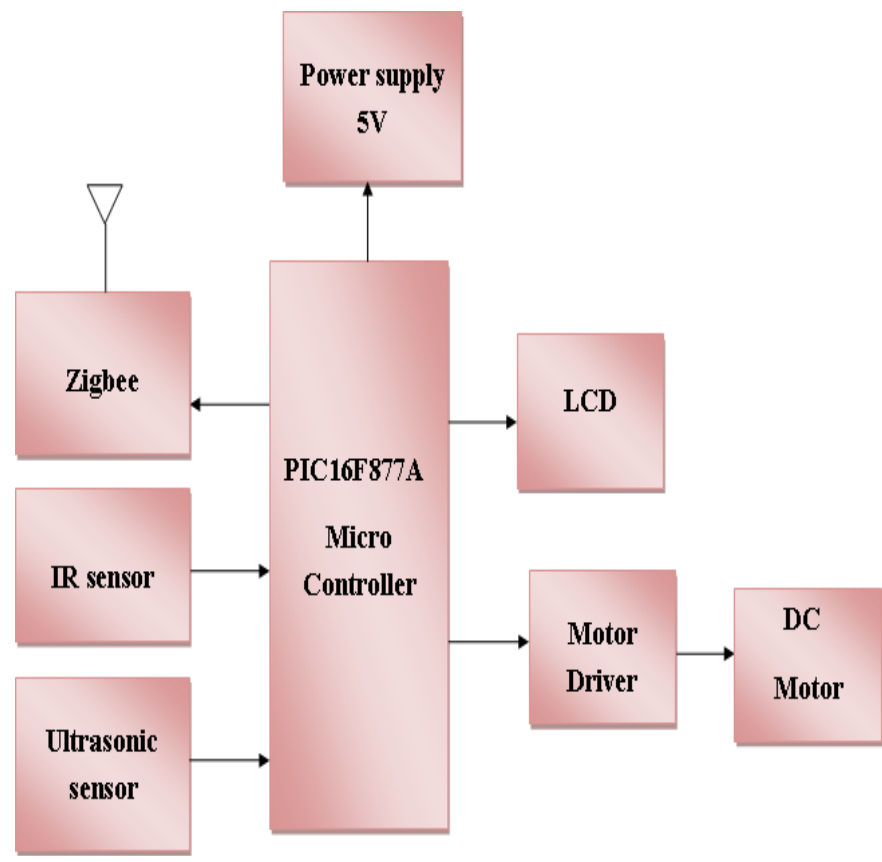

\section{Server Section:}

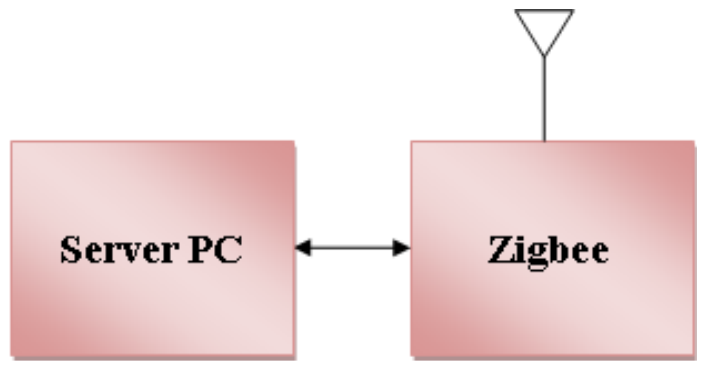


Bus to House: Voice \& Touch Based Dynamic Bus Navigation System Based On Zigbee, Iot \& ..

\begin{tabular}{|c|c|c|c|}
\hline $\begin{array}{l}\text { Technology/ } \\
\text { feature }\end{array}$ & Example & Contributions & \\
\hline Hardware & $\begin{array}{l}\text { Google } \\
\text { Mercedes-Benz, } \\
\text { BMW, Audi, }\end{array}$ & $\begin{array}{l}\text { Demonstrate the feasibility of } \\
\text { AVs } \\
\text { Show the confidence of the in- } \\
\text { dustry in AVs }\end{array}$ & 4] \\
\hline Software & $\begin{array}{l}\text { Mladenovic \& } \\
\text { Abbas } \\
\\
\text { Petrov \& } \\
\text { Nashashibi }\end{array}$ & $\begin{array}{l}\text { Enhance self-organizing and co- } \\
\text { operative control of AVs } \\
\text { Balance the efficiency and } \\
\text { pafety of AVs } \\
\text { Enhance self-control of AVs }\end{array}$ & 10] \\
\hline system & & $\begin{array}{l}\text { Investigate the scheduling and } \\
\text { admission control problems }\end{array}$ & \\
\hline
\end{tabular}

\section{Zigbee Sensor:}

ZigBee is a wireless technology developed as an open global standard to address the unique needs of low-cost, low-power wireless M2M networks. It operates on the IEEE 802.15.4 physical radio specification and operates in unlicensed bands including $2.4 \mathrm{GHz}, 900 \mathrm{MHz}$ and $868 \mathrm{MHz}$. It is an IEEE 802.15.4-based specification for a suite of high-level communication protocols used to create personal area networks with small, low-power digital radios.

The advantages of Zigbee are: Setting up the network is very simple and easy. It does not have central controller and loads are distributed evenly across the network.

It is easy to monitor and control home appliances from remote.

It will take the place of existing Infrared technology based devices. This will save cost of battery replacement as zigbee uses lithium battery which lasts long. The network is scalable and it is easy to add/remot e zigbee end device to the network.

It requires knowledge of the system for the owner to operate zigbee compliant devices.

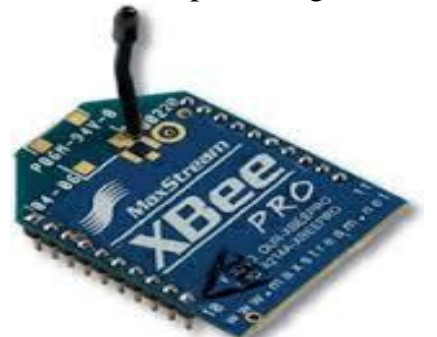

Zigbee protocol architecture consists of a stack of various layers where IEEE 802.15.4 is defined by physical and MAC layers while this protocol is completed by accumulating Zigbee's own network and application layers.

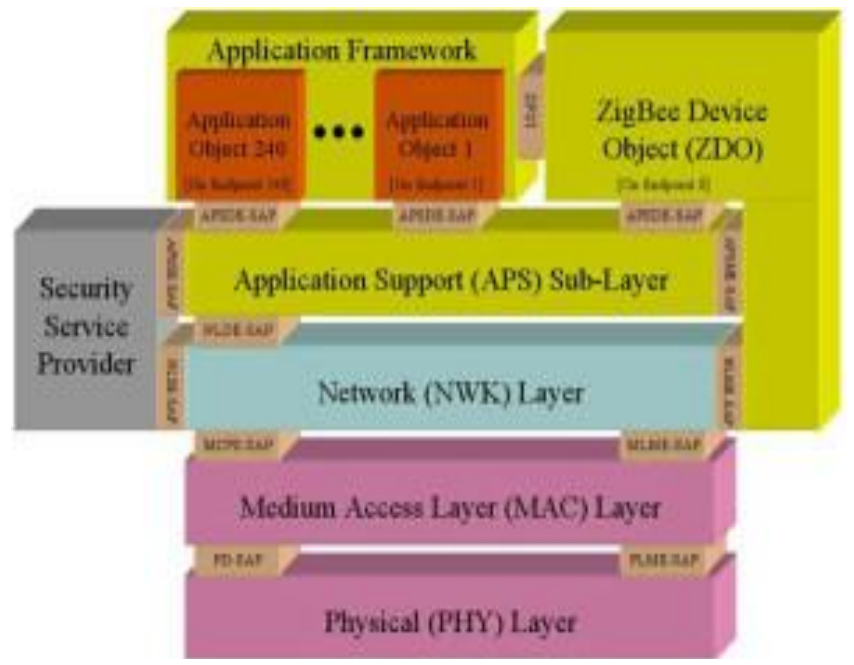




\section{Applications of Zigbee Technology}

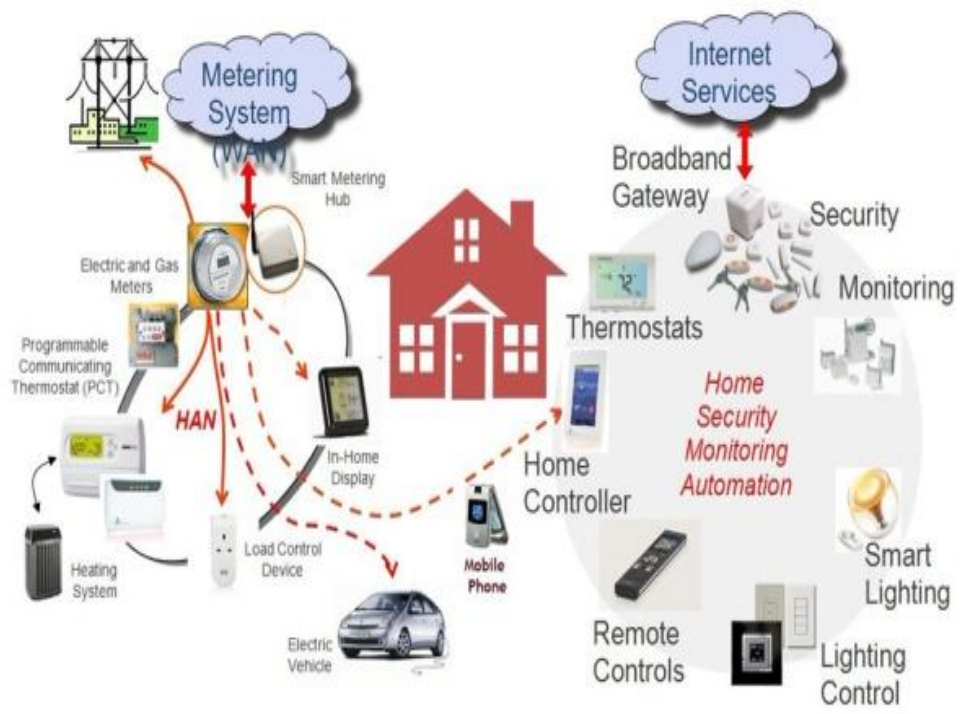

\section{Conclusion}

Thus we are Developing an Android based User Interface for i)Fetching the Nearest available Bus, ii) Its time to reach the Source and iii)The Distance which will surely avoid the waiting time for Android User. The future enhancement can be in the areas of traffic updates. One of our projects major applications is its use as an autonomous $\mathrm{Cab}$ dispatch system. The $\mathrm{Cab}$ dispatch problem involves assigning Cabs to callers waiting at different locations. In such an application our system can be deployed to provide enhanced fuel and time efficiency.

\section{REFERENCES}

[1]. Peter H. Dana. "Global Positioning System Overview." Internet: http://www.colorado.edu/geography/gcraft/notes/gps/gps_f.html, 1994 [Sep. 15,2013].

[2]. Arduino, "Arduino Microcontrollers," arduinoBoardUno datasheet, [Oct. 14.2013].

[3]. Aziz, Mochamad Vicky Ghani, Rifki Wijaya, Ary Setijadi Prihatmanto, and Diotra Henriyan. "HASH MD5 function implementation at 8-bit microcontroller", 2013 Joint International Conference on Rural Information \& Communication Technologyand Electric-Vehicle Technology (rICT \&ICeV-T), [2013].

[4]. Arduino, "Arduino GSM Shield Schematic," GSM shield documentation. Oct. 15.2013].

[5]. "GSM".Internet:http://en.wikipedia.org/wiki/GSM, [Oct. 15.2013].

[6]. Douglas W. Gage (1995). "A Brief History of Unmanned Ground Vehicle (UGV) Development Efforts." Unmanned Systems Magazine [Online]. 13(3), pp. 1-8. Available: www.dtic.mil/cgibin/ GetTRDoc?AD=ADA422845 [Dec. 5,2013].

[7]. Arduino, “Arduino Microcontrollers," arduinoBoard Mega 2560 datasheet http://arduino.cc/en/Main/arduinoBoardMega2560, [Oct. 15.2013].

[8]. "Products for the wireless communications and GNSS:. Internet: http://www.u-blox.com/en/embedded-gps-and-gsm-products.html, [Nov.3,2013]. 